\title{
Variable step direct block multistep method for general second order ODEs
}

\begin{abstract}
Direct block multistep method is developed for the numerical solution of second order ordinary differential equations (ODEs). This method was designed for computing the solution at four points simultaneously using variable step size. The development of this method based on numerical integration and using interpolation approach which are similar to the Adams method. In order to gain an efficient and reliable numerical approximation, this developed block method is implemented in the predictor corrector mode using simple iteration technique. This method has also been proven as a convergence method under suitable conditions of stability and consistency. Several tested problems are taken into account in the numerical experiments and were compared with the existing method. The results obtained showed that this developed block method managed to produce good results.
\end{abstract}

Keyword: Block method; General second order ordinary differential equations; Variable step size 